\title{
The effect of chitosan nanospheres on the immunogenicity of Toxoplasma lysate vaccine in mice
}

\author{
Mona M. El Temsahy $\cdot$ Eman D. H. El Kerdany $\cdot$ Maha M. Eissa • \\ Thanaa I. Shalaby $\cdot$ Iman M. Talaat $\cdot$ Nermine M. F. H. Mogahed
}

Received: 18 May 2014/ Accepted: 22 August 2014/Published online: 5 September 2014

(C) Indian Society for Parasitology 2014

\begin{abstract}
Toxoplasmosis, a zoonotic parasitic disease, is a huge challenge for which there is no effective vaccine up till now. In this study, chitosan nanospheres encapsulated with Toxoplasma lysate vaccine was evaluated for its ability to protect mice against both acute and chronic toxoplasmosis models of infection. Results showed that chitosan nanospheres were equally effective to Freund's incomplete adjuvant (FIA) in enhancing the efficacy of Toxoplasma lysate vaccine. The effectiveness was demonstrated by the delayed death of vaccinated mice following challenge either with virulent $\mathrm{RH}$ or avirulent Me49 strains, the significant decrease in parasite density in different organs, significant increase in the humoral and cellular immune response (IgG and IFN $\gamma$ ) with a marked reduction of pathological changes in the different organs. However chitosan nanospheres were superior to FIA due to their cost effective preparation and much less necrotic changes induced in the studied organs. The success of chitosan polymer as an alternative to commonly used adjuvants paves the way for the use of other newly developed polymers to be used in the field of vaccine development.
\end{abstract}

M. M. El Temsahy · E. D. H. El Kerdany •

M. M. Eissa ( $\square)$ · N. M. F. H. Mogahed

Department of Medical Parasitology, Faculty of Medicine,

University of Alexandria, Alexandria, Egypt

e-mail: mahaeissa19@yahoo.com

T. I. Shalaby

Department of Biophysics and Biomedics, Medical Research Institute, University of Alexandria, Alexandria, Egypt

I. M. Talaat

Department of Pathology, Faculty of Medicine, University of Alexandria, Alexandria, Egypt
Keywords Crude Toxoplasma lysate vaccine . Virulent RH Toxoplasma strain . Avirulent Me49 Toxoplasma strain . Encapsulated chitosan nanospheres

\section{Introduction}

Toxoplasmosis is a zoonotic parasitic disease of great importance for both human and animals (Black and Boothroyd 2000) with worldwide distribution (Tenter et al. 2000). Human infection occurs after consumption of tissue cysts in under cooked meat or from accidental ingestion of oocysts shed in cat's faeces. In most individuals the infection is asymptomatic, whereas severe pathology and lethality are common findings in congenitally infected or immunodeficient individuals (Jongert et al. 2009).

The disease is a huge challenge for which there is no effective treatment up till now (Abu-Madi et al. 2008). Although the current drug therapy can effectively kill the tachyzoite stage, such treatment does not remove the chronic bradyzoite stage (cyst), and thus long-term therapy is needed. The toxic side effects of the drugs, combined with their inability to eliminate the infection, make the need for the development of a vaccine against $T$. gondii infection of a great priority (Black and Boothroyd 2000).

Different trials of vaccination against toxoplasmosis have been conducted mainly in mice using several types of vaccines as, killed Toxoplasma vaccine (Mady 2005), live attenuated whole parasite vaccine (Ismael et al. 2006) and purified parasite antigen vaccine (Martinez-Gomez et al. 2009). Different DNA vaccines mostly originated from dense granules, rhoptries and micronemes were developed. However, the main disadvantage was their inability to induce a sterile immunity against infection with cyst forming T. gondii isolates (Angus et al. 2000). 
Previous studies confirmed that the use of adjuvants increases the efficacy of vaccines by up to $82 \%$ (Palatnikde-Sousa 2008). However, the most effective adjuvants as Freund's adjuvants, generally cause severe inflammation with unacceptable side effects (Handman 2001). With the introduction of nanotechnology into the medical field, efforts to develop a new delivery system for vaccines had been developed. Different trials were done using microspheres and nanospheres in immunization against bacterial, viral and parasitic diseases with promising results (DaneshBahreinni et al. 2011). Nanovaccines are similar to regular vaccine, but they are more advanced. They consist of the same pathogen in synthetic particles instead of solutions. These synthetic nano-particles are prepared from biodegradable and biocompatible polymers (Tiyaboonchai 2003). Chitosan is one of the available water-soluble polymers. It is a modified natural carbohydrate polymer formed by partial N-deacetylation of chitin. It does not only act as an adjuvant, but it can also increase the antigen size and activate the immune system. This indicates that it could be a good adjuvant for a simple antigenic vaccine (Danesh-Bahreinni et al. 2011).

Therefore, the aim of this study was to evaluate the efficacy of chitosan nanospheres as an adjuvant on the immunogenicity of Toxoplasma lysate vaccine as compared to Freund's incomplete adjuvant (FIA) in the mouse model.

\section{Materials and methods}

Parasites

Virulent T. gondii RH HXGPRT(-) strain

RH strain was maintained in the laboratory of the Medical Parasitology Department, Faculty of Medicine, Alexandria University, Egypt by continuous intraperitoneal (IP) passages into laboratory bred Swiss strain albino mice every 3 days (McLeod et al. 1988).

\section{Avirulent T. gondii Me49 strain}

Me49 strain was maintained by oral inoculation of Swiss albino mice each with 20 cysts obtained from brains of previously infected mice 60 days earlier (Hermes et al. 2008). This strain was kindly provided by Professor Dr. Ashraf Barakat, Professor of Epidemiology and Zoonotic Diseases, Epidemiology and Zoonotic Diseases Department, National Research Center, Dokii, Giza, Egypt.
Adjuvants

\section{Blank chitosan nanospheres (BCNS)}

BCNS were prepared according to the method of DaneshBahreinni et al. (2011) and Ravindran et al. (2010) using ionotropic gelation process. Both chitosan (deacetylation $\geq 85 \%$; Sigma) and tripolyphosphate pentabasic (TPP) powder (Sigma-Aldrich) were used in the preparation of the nanospheres. Briefly, $80 \mathrm{mg}$ of TPP were added to $40 \mathrm{ml}$ of distilled water in a glass beaker. In another one, $28 \mathrm{mg}$ of chitosan powder were dissolved in $40 \mathrm{ml}$ of $1 \%$ acetic acid glacial using a magnetic stirrer at room temperature. TPP solution was added at once on the dissolved chitosan solution. The $\mathrm{pH}$ of the solution was adjusted to 6.5 followed by continuous stirring for $1 \mathrm{~h}$ then centrifuged at $12,000 \mathrm{rpm}$ for $15 \mathrm{~min}$ at room temperature. The sediment was collected, lyophilized and the actual weight was measured.

FIA: was purchased from (Sigma, Lot No. 051M872).

\section{Vaccines}

\section{Crude Toxoplasma lysate vaccine (CTLV)}

Tachyzoites were collected from the peritoneal cavity of previously infected mice. They were sedimented by centrifugation at 2,000 rpm for $10 \mathrm{~min}$, washed three times in PBS, then sonicated for eight cycles each for $10 \mathrm{~s}$ with $5 \mathrm{~s}$ interval in between under cooling conditions $\left(4^{\circ} \mathrm{C}\right)$. Toxoplasma lysate vaccine was centrifuged at $1,000 \mathrm{rpm}$ for $20 \mathrm{~min}$. The supernatant material (the lysated antigen of Toxoplasma) was collected and preserved at $-20{ }^{\circ} \mathrm{C}$. Protein concentration was determined by using a commercial total protein determination kit (Turner 1983; Yap et al. 1998; Khan and Casciotti 1999).

\section{CTLV encapsulated chitosan nanospheres (CTLVECNS)}

Preparation of CTLVECNS was done as previously described except that TPP was dissolved in $40 \mathrm{ml}$ of thawed Toxoplasma lysate vaccine, instead of the distilled water. The solution was centrifuged at $12,000 \mathrm{rpm}$ for $15 \mathrm{~min}$. The sediment was collected; lyophilized and weighed. The total amount of protein per mg of chitosan encapsulating powder was determined by dividing the protein concentration of the loaded vaccine by the weight of nanospheres. Each one $\mathrm{mg}$ of chitosan nanospheres powder was adjusted to contain $1 \mathrm{mg}$ of the vaccine protein (Danesh-Bahreinni et al. 2011; Ravindran et al. 2010).

The loading efficiency (LE) of the prepared nanospheres was calculated according to the following formula: 
$\% \mathrm{LE}=\frac{\mathrm{A}-\mathrm{B}}{\mathrm{A}} \times 100$,

A is the total protein concentration of Toxoplasma lysate vaccine, $\mathrm{B}$ is the concentration of proteins in supernatant.

The \% LE of the prepared nanospheres was $66.6 \%$ (from each $1.5 \mathrm{mg}$ Toxoplasma lysate vaccine protein added, only $1 \mathrm{mg}$ was encapsulated within the nanoparticles).

\section{Characterization of nanoparticles}

Laser defraction particle size analyzer (Zetasizer, Nano, Malvern Co., UK) was used to measure the diameter and the size distribution of the nanoparticles. Morphological examination was performed by both scanning electron microscope (SEM; JEOL, JSM 6360 LA, Japan) and transmission electron microscope (TEM; JEOL-100CX). The X-ray diffraction (XRD) pattern of both blank and vaccine encapsulated were recorded on an X-ray diffractometer (Shimadzu, XRD-700, Maxima, Japan) (DaneshBahreinni et al. 2011; Tafaghodi et al. 2008).

Preparation of vaccine for immunization of mice

$5 \mathrm{mg}$ of lyophilized CTLVECNS were suspended in $10 \mathrm{ml}$ of sterile PBS, followed by further dilution so, each mouse received $20 \mu \mathrm{g}$ of CTLVECNS in $100 \mu \mathrm{l}$ of PBS by IP route.

Animal grouping and vaccination schedule

The experimental protocol followed the guidelines for the care and use of laboratory animals authorized by Ethics Committee, Faculty of Medicine, Alexandria University, based on international regulations of animal care. Experiments were carried out on 200 male Swiss strain albino mice, $6-8$ weeks old. They were purchased from the animal house of the Medical Parasitology Department, Faculty of Medicine, Alexandria University, Egypt. Mice were divided into two main groups, Group I control group (100 mice) which was further subdivided into five equal subgroups (20 mice) subgroup Ia: normal non infected, subgroup Ib: chitosan delivery control subgroup, received $20 \mu \mathrm{g}$ of BCNS in $100 \mu \mathrm{l}$ of PBS/mouse/dose, subgroup Ic: FIA control subgroup, received $100 \mu \mathrm{l}$ of FIA/mouse/dose, subgroup Id: RH infected control subgroup, infected by 2,500 tachyzoites and Ie: Me49 infected control subgroup, perorally inoculated by 10 cysts of Me49 strain. Group II experimental vaccinated group (100 mice) was further subdivided into two equal subgroups (50 mice). Subgroup IIa, received CTLVECNS suspended in PBS while subgroup IIb received CTLV + FIA. All vaccinated mice received three doses 2 weeks apart. Mice of subgroup IIa1 and IIb1 were challenged 4 weeks after the last vaccination dose by 2,500 viable tachyzoites of virulent RH strain injected IP (Araujo et al. 1992), while mice of subgroups IIa2 and IIb2 were challenged orally by 10 cysts of Me49 strain (Krahenbuhl et al. 1972). In mice vaccinated and challenged with virulent RH strain, 10 mice out of 25 were sacrificed 5 days post infection and the remaining mice were observed daily to record mortality. On the other hand, in vaccinated mice challenged with avirulent Me49 strain, 10 mice out of 25 were sacrificed 60 days post infection and the remaining were observed daily to record mortality. Serum samples were collected from RH and Me49 infected control subgroups mice before being sacrificed simultaneously with their corresponding experimental subgroups.

Assessment of vaccine efficacy

Parasitological study,

Survival time,

Daily observation of mice was done to record the mortality.

\section{Estimation of the parasite count}

Counting of the parasite (tachyzoites) was done in Giemsa stained impression smears of the liver, the spleen, the mesenteric lymph nodes (MLNs) and the brain of mice of virulent RH infected studied subgroups. The mean of 10 different oil immersion fields from each studied organ of each mouse was calculated then the mean of the subgroup was determined (Thiptara et al. 2006; Garcia et al. 2005). Counting of the Toxoplasma cysts was done in tissue sections of the brain stained with haematoxylin and eosin (H\&E) for mice of avirulent Me49 infected studied subgroups. The mean of 10 different high power fields from each mouse was calculated then the mean of the subgroup was determined (Araujo et al. 1992).

\section{Histopathological study}

Specimens from the liver, the spleen, the MLNs and the brain tissues were fixed in $10 \%$ neutral formalin, embedded in paraffin then serial sections, $5 \mu \mathrm{m}$ thick, were cut using microtome and processed for staining using H\&E stain (Drury and Wallington 1980).

Immunological studies

Interferon gamma (IFN $\gamma$ ), anti-Toxoplasma $\operatorname{IgG}$ and antiToxoplasma IgM levels were measured in serum samples (Stoicov et al. 2004; Martinez-Gomez et al. 2009). 
Determination of IFN $\gamma$ level in sera of sacrificed mice was done using ELISA kit (KOMA Kit, Biotech, Inc.; Stoicov et al. 2004).

Determination of anti-Toxoplasma IgG/IgM levels in sera of sacrificed mice was measured using ELISA kits for detection of anti-Toxoplasma IgG/IgM (WKEAMED supplies; Martinez-Gomez et al. 2009).

\section{Statistical analysis}

Data were fed to the computer and analyzed using IBM SPSS software package version 20.0 (Kirkpatrick and Feeney 2013). Quantitative data were described using mean and standard deviation, median, minimum and maximum (Leslie et al. 1991).

The distributions of abnormally distributed data, comparison between two independent populations were done using Mann-Whitney test (Chan 2003), while \% reduction was calculated according to the following formula:
Parasitological study

Survival time: none of the mice of RH infected control subgroup remained alive beyond the seventh day post infection with a mean survival time of $5.9 \pm 0.88$ days (Fig. 2). On the other hand, in the avirulent Me49 infected control subgroup, the mean survival time was $60.7 \pm 8.11$ days with only one mouse out of 10 which lived for 120 days post infection (Fig. 3). Animals vaccinated with CTLV either in chitosan nanospheres or in combination with FIA as an adjuvant and challenged with virulent RH strain showed significant increase in their survival time with a maximum survival time of 80 days with a mean of $35.73 \pm 31.82$ and 57 days with a mean of $33.67 \pm 18.30$ days post infection, respectively (Fig. 2). As regards the Me49 strain, only mice vaccinated with CTLV in combination with FIA showed a statistically significant increase in the survival time when compared to the corresponding control. There was a statistically significant dif-

Mean of the vaccinated subgroup - Mean of the control subgroup

Mean of the control subgroup

$\times 100$

\section{Results}

Nanospheres characterization

By SEM, dried nanospheres either blank or vaccine encapsulated showed regular spherical shapes with a smooth surface. The mean size of BCNS and CTLVECNS were $182.024 \pm 42.07481$ and $211.896 \pm 67.5812 \mathrm{~nm}$, respectively, indicating enlargement of the nanospheres after vaccine encapsulation (Fig. 1a, b).

TEM of partially hydrated nanospheres showed their spherical, distinct and regular shapes. The surface was smooth in BCNS and rough in CTLVECNS. The mean size of BCNS and CTLVECNS were 180.67 \pm 33.71 and $311 \pm 48.28 \mathrm{~nm}$, respectively and the size distribution between them was quite narrow (Fig. 1c-f).

The average hydrated particle size of CTLVECNS was $221.5 \mathrm{~nm}$ as measured by Zetasizer instrument with a quite narrow monomodal size distribution.

Three prominent crystalline peaks at $31.6^{\circ}(2 \theta)$ and $64.3^{\circ}(2 \theta)$ and $77.4^{\circ}(2 \theta)$ for BCNS and at $44^{\circ}(2 \theta)$ and $64.2^{\circ}(2 \theta)$ and $77.4^{\circ}(2 \theta)$ for CTLVECNS were observed using X-ray diffractometer (XRD) indicating stability of the encapsulated nanospheres. ference between mice vaccinated with CTLV whether in chitosan nanospheres or in combination with FIA with a mean level of $79.20 \pm 17.91$ and $120.53 \pm 33.13$, respectively (Fig. 3).

Parasite count

Toxoplasma gondii tachyzoites were detected in Giemsa stained impression smears from the liver, the spleen, the MLNs and the brain of animals of both virulent $\mathrm{RH}$ infected control subgroup and both vaccinated RH challenged subgroups (chitosan nanospheres vaccinated and FIA vaccinated subgroups). There was a statistically significant reduction in parasitic count in all studied organs in vaccinated animals as compared to their corresponding control. However this reduction in the mean tachyzoites count was found to be more evident in the subgroup receiving encapsulated chitosan vaccine in comparison to the subgroup receiving the vaccine in combination with FIA (Table 1).

Regarding mice challenged with Me49 strain, a statistically significant reduction in the mean cyst count of the brain was found in both experimental subgroups either receiving encapsulated chitosan vaccine or receiving the 

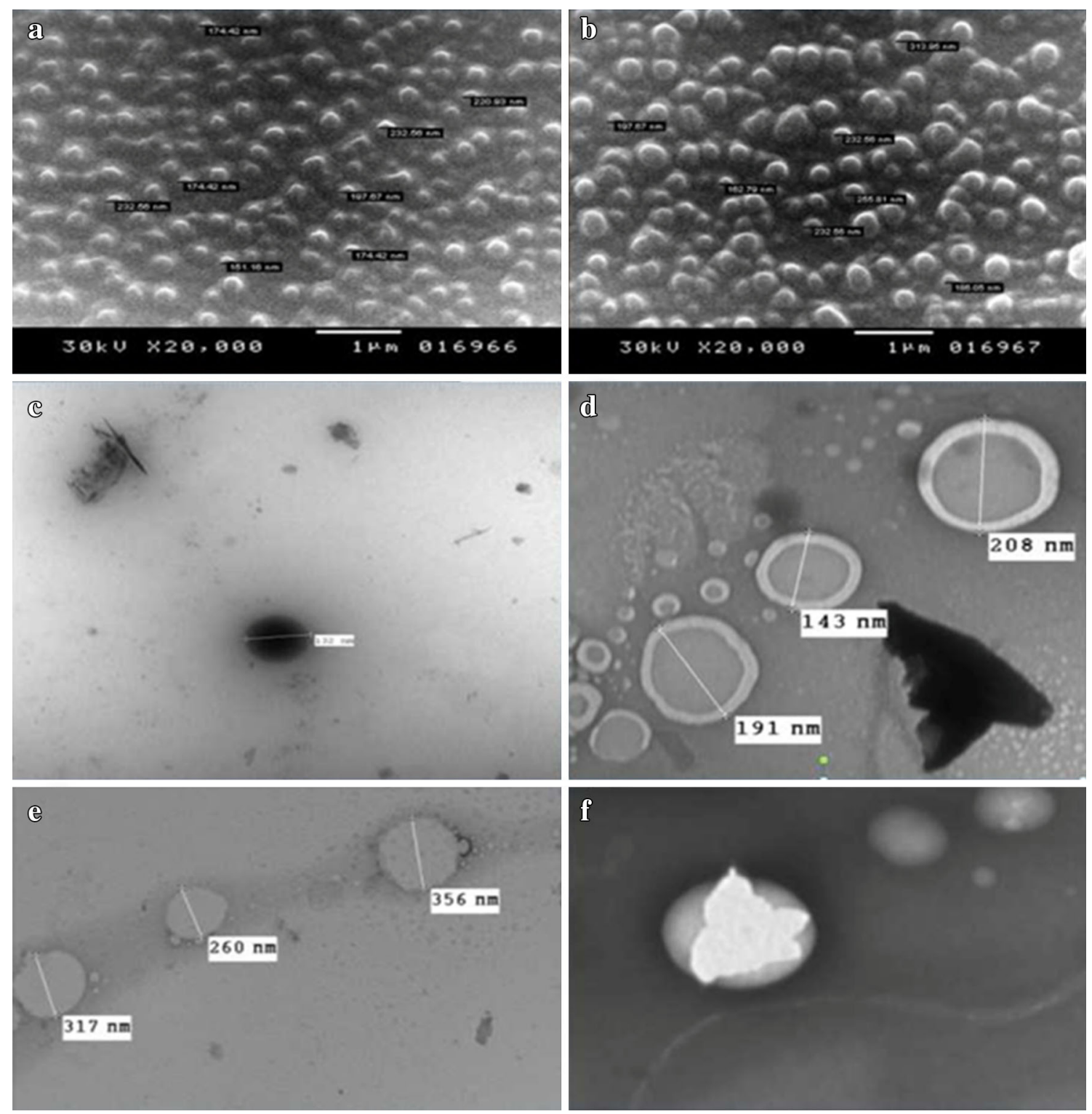

Fig. 1 Scanning and transmission electron microscope of both BCNS

$(\times 3,000)$. d TEM of BCNS showing double membrane nanospheres and CTLVECNS. a SEM of BCNS showing spherical shaped nanospheres with smooth external surface $(\times 20,000)$. b SEM of CTLVECNS showing enlarged nanospheres $(\times 20,000)$. c TEM of BCNS showing spherical shaped nanospheres with smooth surface $(\times 10,000)$. e TEM of CTLVECNS showing spherical nanospheres with rough surface $(\times 3,000)$. f TEM of CTLVECNS showing spherical nanospheres with encapsulated antigen $(\times 10,000)$

vaccine in combination with FIA when compared to their corresponding control. The difference between both vaccinated subgroups was statistically significant with the highest reduction in the subgroup receiving the vaccine in combination with FIA (Table 2).

\section{Histopathological study}

\section{Liver}

Normal liver tissue showed polygonal mature hepatocytes with rounded central nuclei. The hepatocytes were disposed in two

cell thick trabeculae radiating from central veins peripherally. Portal tracts were seen containing a branch from the hepatic artery, a tributary from the hepatic vein and a bile ductule. No inflammatory cellular infiltrates were observed (Fig. 4a). Animals injected with BCNS alone showed mild-moderate infiltration of the portal tracts by chronic inflammatory cells in addition to dilation and congestion of the vascular spaces (Fig. 4b). In FIA control subgroup foci of lytic necrosis (Fig. 4c), open disturbed chromatin as well as binucleated cells with increase in the nucleocytoplasmic ratio were observed (Fig. 4d).

Animals infected with the virulent RH strain showed ballooning of the hepatocytes, fatty degenerative changes 


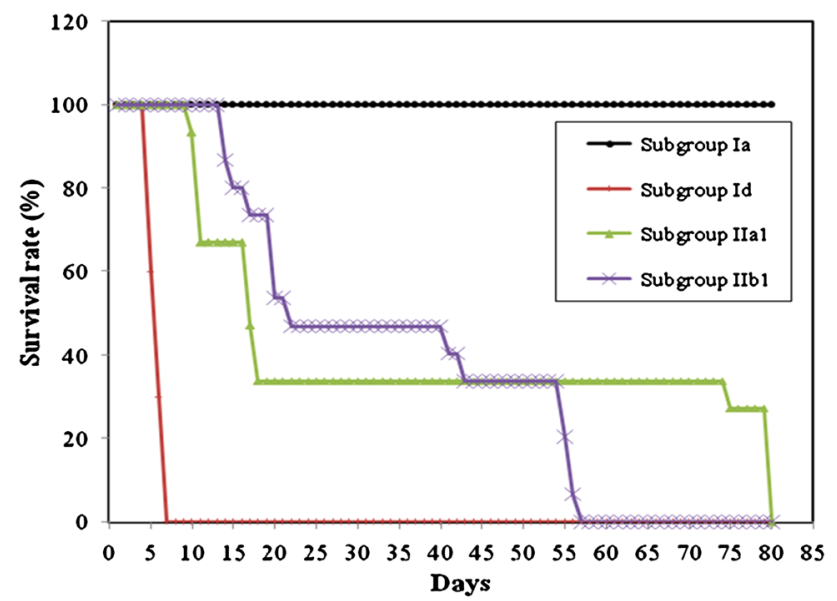

Fig. 2 The survival time of different RH infected subgroups. (Ia) Normal non infected control subgroup (Id) virulent $\mathrm{RH}$ infected control subgroup (IIal): CTLVECNS vaccinated subgroup challenged with virulent RH strain. (IIbl) CTLV + FIA vaccinated subgroup challenged with virulent RH strain

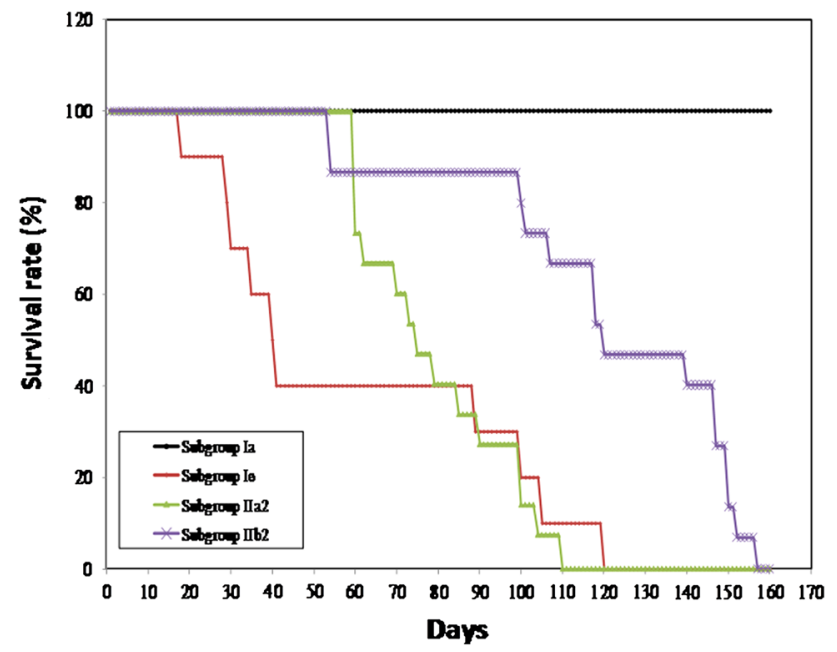

Fig. 3 The survival time of different Me49 infected subgroups. (Ia) normal non infected control subgroup. (Ie) Avirulent Me49 infected control subgroup. (IIa2) CTLVECNS vaccinated subgroup challenged with avirulent Me49 strain. (IIb2) CTLV + FIA vaccinated subgroup challenged with avirulent Me49 strain

(Fig. 4e), severe degree of lymphocytic inflammatory infiltrates within the portal tracts as well as multiple foci of lytic necrosis and interface hepatitis (Fig. 4f). Toxoplasma tachyzoites within the hepatocytes were also detected (Fig. 4g). On the other hand, those vaccinated with CTLV either within chitosan nanospheres or in combination with FIA showed reduction in the severity and extent of inflammatory infiltrates with very few foci of lytic necrosis within the parenchyma of liver cells (Fig. 4h). The reduction in the severity of inflammation was more prominent in the subgroup receiving chitosan encapsulated vaccine when compared to the subgroup receiving the vaccine in combination with FIA (Fig. 4i).
Spleen

Mice infected with the virulent RH strain showed hyperplastic lymphoid follicles, dilated congested sinusoids and multinucleated giant cells indicating severe degree of inflammatory changes (Fig. 5a). Toxoplasma pseudocyst containing tachyzoites were also seen (Fig. 5b).

Mice vaccinated with chitosan encapsulated vaccine showed reactive lymphoid follicles changes with no evidence of necrotic changes (Fig. 5c). The same quality of reactive changes was seen in the FIA vaccinated subgroup but to a lesser degree with evident multinucleated giant cells in the red pulp (Fig. 5d).

\section{$M L N s$}

Mice infected with virulent RH strain showed lymphoid hyperplasia (Fig. 6a) with a prominent germinal center infiltrated with tachyzoites (Fig. 6b). A mild degree of lymphoid hyperplasia was noticed in chitosan vaccinated subgroup but no tachyzoite infiltrate could be seen (Fig. 6c). Similar changes were noticed in FIA vaccinated subgroup with widening of the paracortical areas (Fig. 6d).

\section{Brain}

In the virulent $\mathrm{RH}$ infected control subgroup, heavy inflammatory infiltrates were noticed in the cortex (Fig. 7a) which was infiltrated by a pseudocyst of Toxoplasma (Fig. 7b). In both vaccinated RH challenged subgroups mild increase in brain cellularity was observed. No inflammatory infiltrates could be detected in chitosan vaccinated subgroup (Fig. 7c), while in FIA vaccinated subgroup, mild lymphocytic infiltration and oedematous changes were detected (Fig. 7d).

Regarding avirulent Me49 infected control subgroup, Toxoplasma cysts were seen surrounded by chronic inflammatory cells, mainly lymphocytes, as well as few histiocytes (Fig. 8a, b). On the other hand, chitosan vaccinated subgroup showed fewer number of brain cysts with mild increase in the brain cellularity and no inflammatory cellular infiltrate (Fig. 8c, d). The same changes were evident in the subgroup receiving the vaccine in combination with FIA with mild oedematous changes (Fig. 8e, f).

Immunological study

$I F N \gamma$

All studied subgroups showed a statistically significant increase in the level of IFN $\gamma$ as compared to the normal non infected control subgroup. A statistically significant increase in the level of IFN $\gamma$ was noticed in the vaccinated 

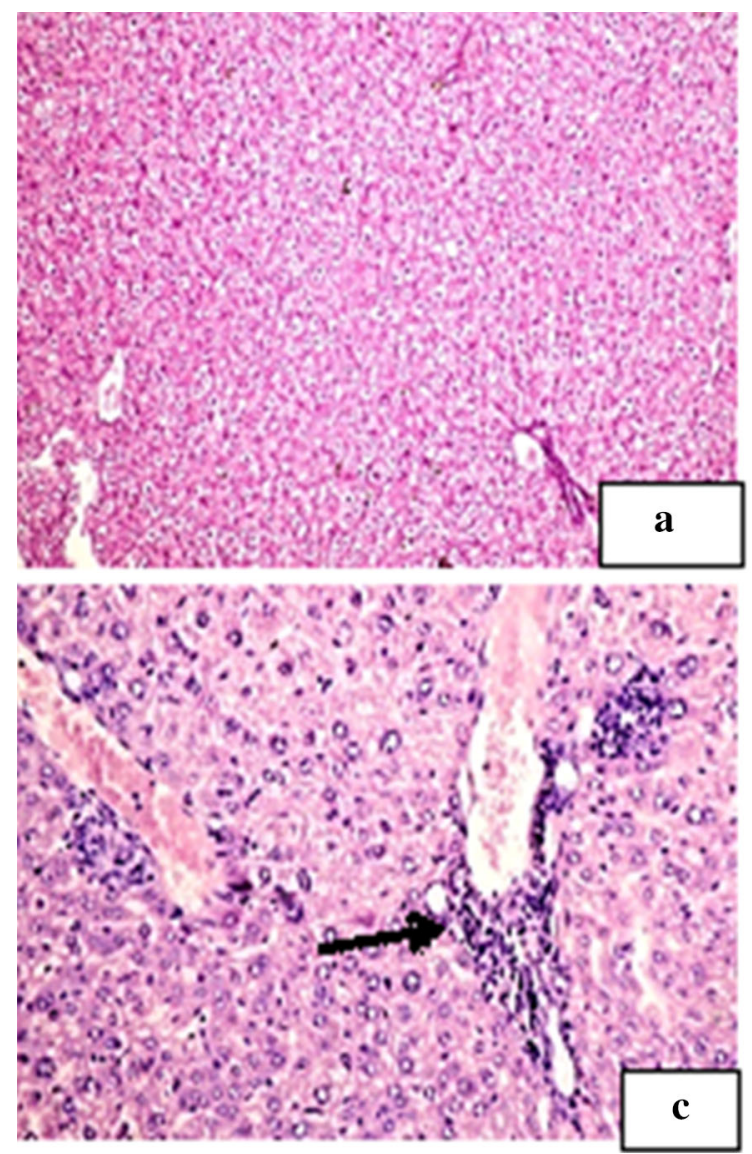

Fig. 4 Liver section of the studied subgroups. a Liver section of normal non infected control subgroup (Ia) showing features of a normal hepatic architecture $(\mathrm{H} \& \mathrm{E} \times 100)$. b Liver section of chitosan delivery control subgroup (Ib) showing mild-moderate infiltration of the portal tract by chronic inflammatory cells $(H \& E \times 200)$. c Liver section of Freund's adjuvant receiving subgroup (Ic) showing periportal tract infiltration with inflammatory cells mainly lymphocytes with a focus of lytic necrosis $(\rightarrow, H \& E \times 200)$. d Liver section of FIA control subgroup (Ic) showing hepatocytes with open nuclei and disturbed chromatin, as well as binucleated cells $(\mathrm{H} \& \mathrm{E} \times 400)$. Liver section of the studied subgroups. e Liver section of virulent RHinfected subgroup (Id) showing ballooning of the hepatocytes and fatty degenerative changes. Mild inflammatory infiltrate is seen

RH challenged subgroups either chitosan encapsulated or in combination with FIA as compared to their corresponding control with no statistically significant difference between them. While in avirulent Me49 Toxoplasma strain infected subgroups, though there was increase in IFN $\gamma$ level in the vaccinated challenged subgroups, yet no statistically significant difference was observed either with their corresponding control subgroup or between the two experimental subgroups (Table 3).

\section{Anti-Toxoplasma $\operatorname{Ig} G$}

As regards infected control subgroups (RH and Me49) both showed a statistically significant increase in the level of
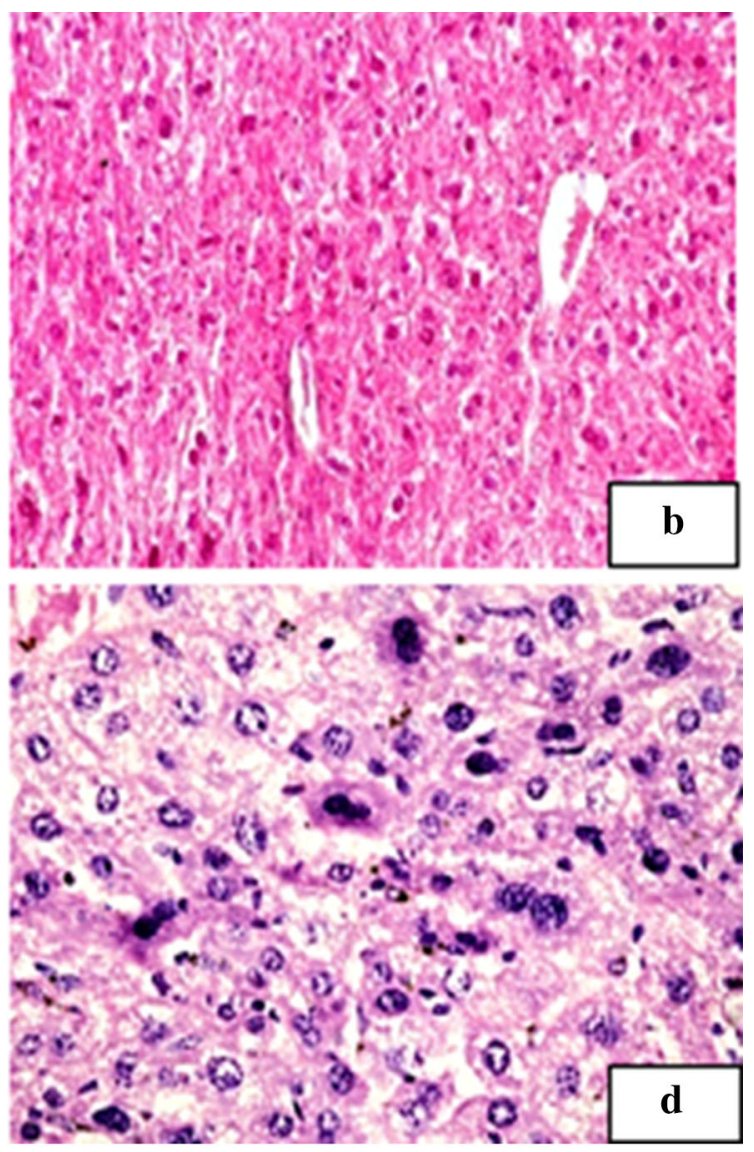

surrounding the portal tract with foci of lytic necrosis $(\rightarrow, \mathrm{H} \& \mathrm{E}$ $\times 200$ ). f Liver section of virulent RH-infected subgroup (Id) showing necrotic zone $(\rightarrow)$ separating normal hepatocytes from inflammatory cellular infiltration $(\mathrm{H} \& \mathrm{E} \times 200)$. g Liver section of virulent RHinfected subgroup (Id) showing $T$. gondii tachyzoites within hepatocyte $(\rightarrow, H \& E \times 1,000)$. h Liver section of CTLVECNS vaccinated subgroup challenged with virulent RH strain (IIa1) showing mild inflammatory infiltrate within the portal tract with few foci of lytic necrosis $(\rightarrow, H \& E \times 100)$. i Liver section of CTLV + FIA vaccinated subgroup challenged with virulent RH strain (IIb1) showing mildmoderate inflammation with minimal interface hepatitis $(\rightarrow, \mathrm{H} \& \mathrm{E}$ $\times 200$ )

IgG in comparison to normal non infected control. A statistically significant increase in the level of $\operatorname{IgG}$ was also noticed in both vaccinated challenged subgroups in comparison to their corresponding infected control subgroups both in acute and chronic infection models (Table 4). There was no statistically significant difference between the two vaccinated subgroups (Table 4).

\section{Anti-Toxoplasma IgM}

No statistically significant increase was detected in $\mathrm{RH}$ infected control subgroup when compared to the normal non infected subgroup. While in Me49 subgroup a statistically significant increase was noted. Both types of 

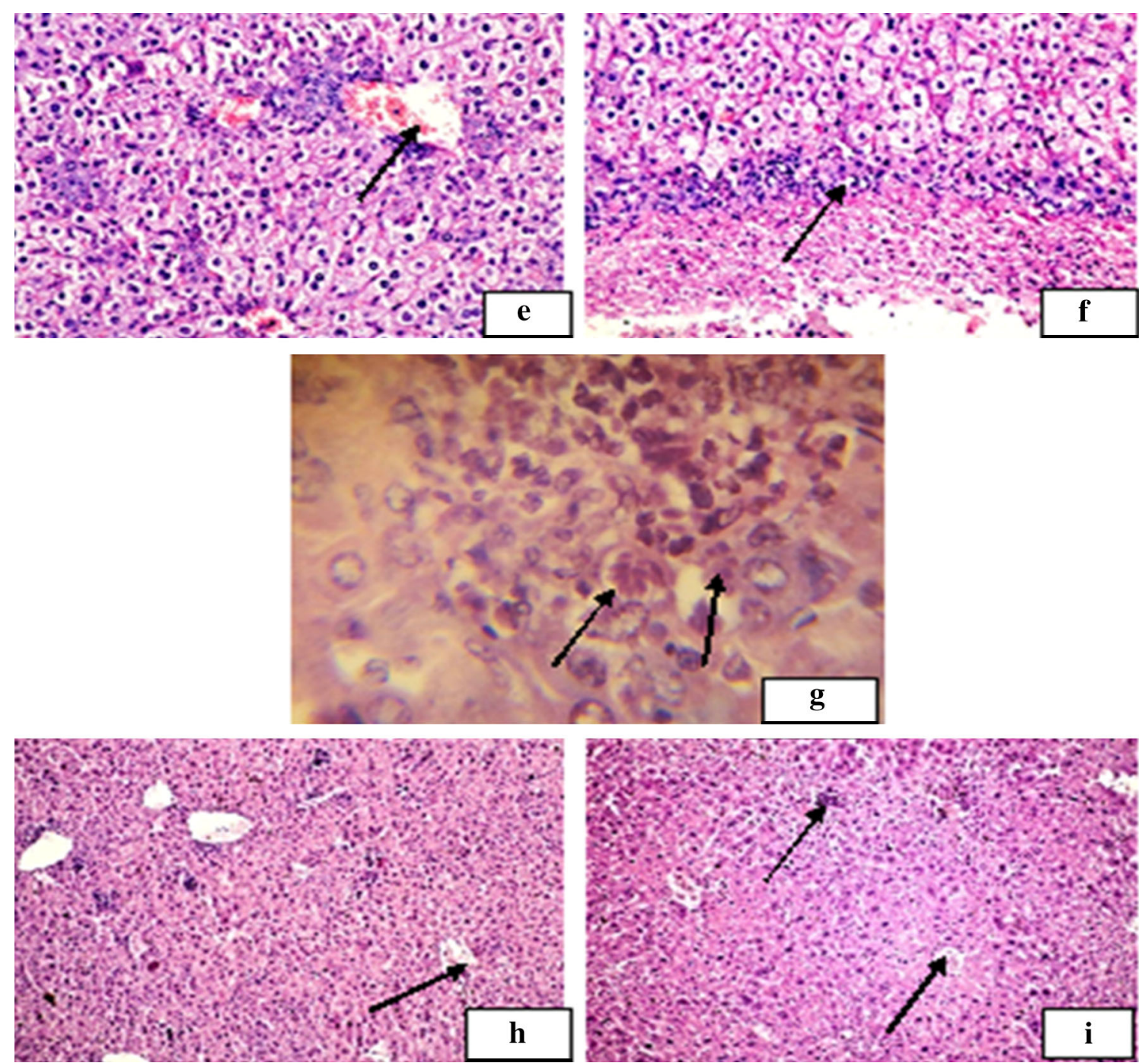

Fig. 4 continued

Table 1 Mean parasitic count and percentage reduction in the different organs in RH infected subgroups

\begin{tabular}{|c|c|c|c|c|c|}
\hline Groups & & Liver & Spleen & MLNs & Brain \\
\hline Group Ia & & $0.0 \pm 0.0$ & $0.0 \pm 0.0$ & $0.0 \pm 0.0$ & $0.0 \pm 0.0$ \\
\hline Group Id & & $7.33 \pm 4.70$ & $2.21 \pm 2.62$ & $5.32 \pm 3.65$ & $1.09 \pm 1.19$ \\
\hline Group IIa1 & & $0.26 \pm 0.14$ & $0.09 \pm 0.10$ & $0.08 \pm 0.11$ & $0.04 \pm 0.10$ \\
\hline Group IIb1 & & $0.98 \pm 0.91$ & $0.86 \pm 1.19$ & $0.94 \pm 1.01$ & $0.15 \pm 0.18$ \\
\hline $\mathrm{p}_{1}$ & Id-IIa1 & $<0.001^{*}$ & $0.001 *$ & $<0.001^{*}$ & 0.076 \\
\hline $\mathrm{p}_{2}$ & Id-IIb1 & $0.002 *$ & 0.102 & $0.005^{*}$ & 0.312 \\
\hline $\mathrm{p}_{3}$ & IIa1-IIb1 & $0.020 *$ & 0.098 & $0.017 *$ & 0.120 \\
\hline$\% \mathrm{r}_{1}$ & & 96.5 & 95.9 & 98.5 & 96.3 \\
\hline$\% \mathrm{r}_{2}$ & & 86.6 & 61.1 & 82.3 & 86.2 \\
\hline
\end{tabular}

Ia normal non infected control subgroup, Id virulent RH infected control subgroup, IIal CTLVECNS vaccinated subgroup challenged with virulent RH strain, IIbl CTLV + FIA vaccinated subgroup challenged with virulent RH strain, \% $r_{l}$ percentage of reduction in chitosan vaccinated subgroup, $\% r_{2}$ percentage of reduction in FIA vaccinated subgroup, $p_{1} p$ value for Mann-Whitney test between subgroup Id and IIa1, $p_{2} p$ value for Mann-Whitney test between subgroup Id and IIb1, $p_{3} p$ value for Mann-Whitney test between subgroup IIa1 and IIb1

* Statistically significant at $p \leq 0.05$ 
Table 2 Mean parasitic count and percentage reduction in the brain in Me49 infected subgroups

\begin{tabular}{|c|c|c|}
\hline Group Ia & & $0.0 \pm 0.0$ \\
\hline Group Ie & & $11.22 \pm 4.92$ \\
\hline Group IIa2 & & $6.0 \pm 3.92$ \\
\hline Group IIb2 & & $2.60+0.98$ \\
\hline $\mathrm{p}_{1}$ & Ie-IIa2 & $0.016^{*}$ \\
\hline $\mathrm{p}_{2}$ & Ie-IIb2 & $0.002 *$ \\
\hline $\mathrm{p}_{3}$ & IIa2-IIb2 & $0.011^{*}$ \\
\hline$\% \mathrm{r}_{1}$ & & 46.5 \\
\hline$\% \mathrm{r}_{2}$ & & 76.8 \\
\hline
\end{tabular}

Ia normal non infected control subgroup, Ie avirulent Me49 infected control subgroup, IIa2 CTLVECNS vaccinated subgroup challenged with avirulent Me49 strain, IIb2 CTLV + FIA vaccinated subgroup challenged with avirulent Me49 strain, $\% r_{l}$ percentage of reduction in chitosan vaccinated subgroup, $\% r_{2}$ percentage of reduction in FIA vaccinated subgroup, $p_{1} p$ value for Mann-Whitney test between subgroup Ie and IIa2, $p_{2} p$ value for Mann-Whitney test between subgroup Ie and IIb2, $p_{3} p$ value for Mann-Whitney test between subgroup IIa2 and IIb2

* Statistically significant at $p \leq 0.05$
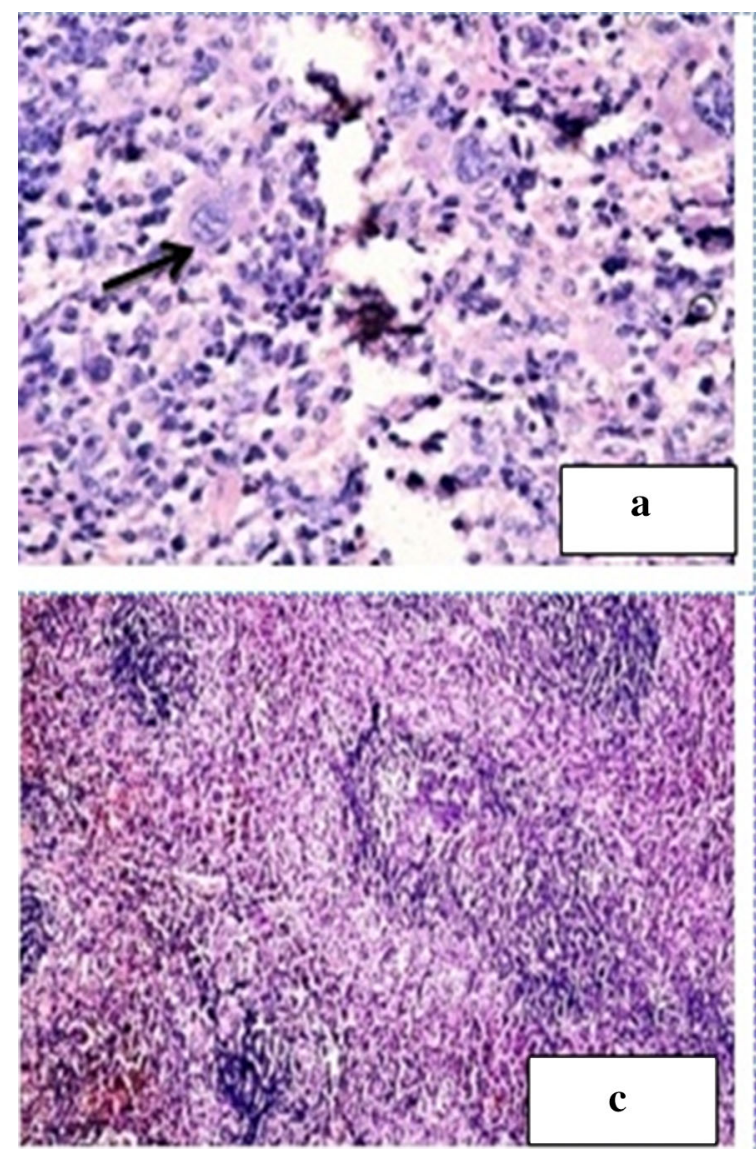

Fig. 5 Spleen sections in the studied subgroups. a Spleen section of virulent RH-infected subgroup (Id) showing mono and multinucleated giant cells in the sinusoids (red pulp, $\rightarrow, \mathrm{H} \& \mathrm{E} \times 400$ ). b Spleen section of virulent RH-infected subgroup (Id) showing Toxoplasma pseudocysts containing tachyzoites $(\rightarrow, \mathrm{H} \& \mathrm{E} \times 1,000)$. c Spleen section of CTLVECNS vaccinated subgroup challenged with virulent vaccine, either encapsulated in chitosan or in combination with FIA, caused a statistically non significant increase in anti-Toxoplasma IgM antibody level either in acute or chronic infection models in comparison to their corresponding control subgroup (Table 5).

\section{Discussion}

In this study multi-antigenic CTLV encapsulated in chitosan nanospheres was evaluated against both acute and chronic toxoplasmosis. Results showed that chitosan nanospheres were able to enhance the immunogenicity of CTLV as demonstrated by increase in the survival time, reduced pathological changes in different organs and enhancement of both cellular and humoral immune response. Chitosan polymer was used in preparation of the nanospheres as its biodegradability, immunological activity and high viscosity make it an excellent candidate as a depot adjuvant for parenteral vaccination (Zaharoff et al. 2007). The immunogenic
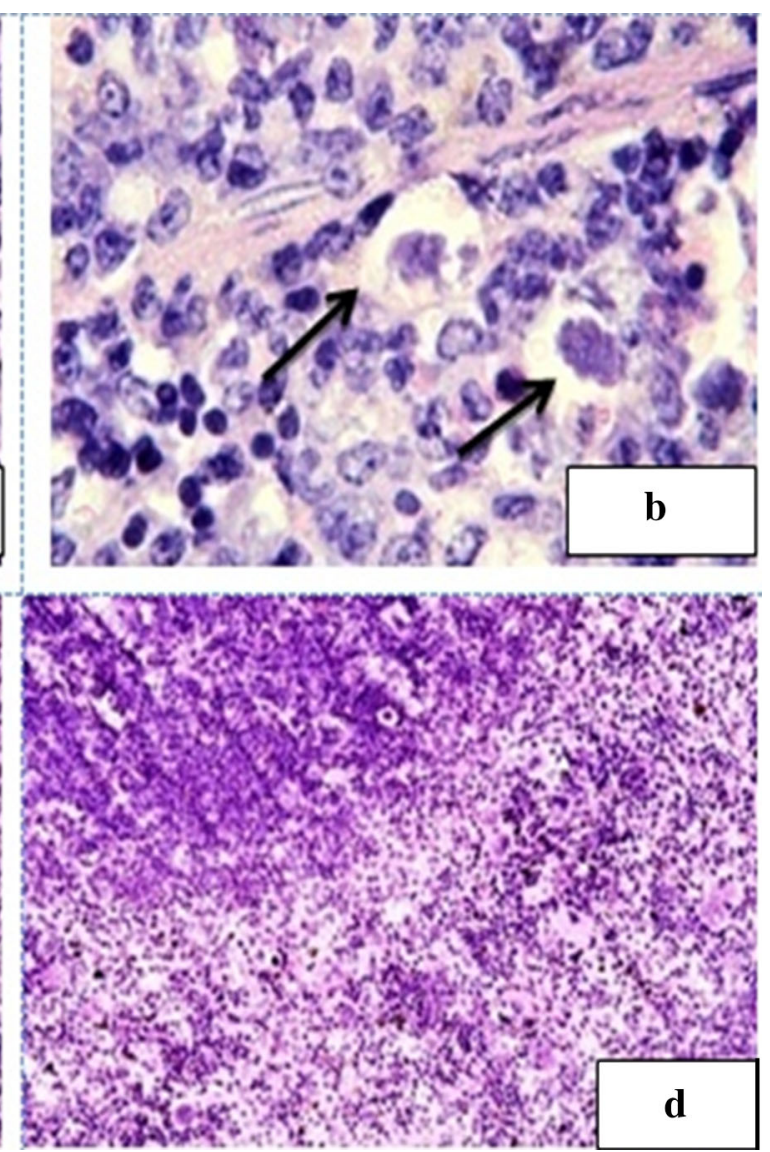

RH strain (IIa1) showing hyperactive lymphoid follicles and dilated congested sinusoids $(H \& E \times 100)$. d Spleen section of CTLV + FIA vaccinated subgroup challenged with virulent RH strain (IIb1) showing parts of lymphoid follicles and dilated congested sinusoids $(\mathrm{H} \& \mathrm{E} \times 100)$. (Color figure online) 


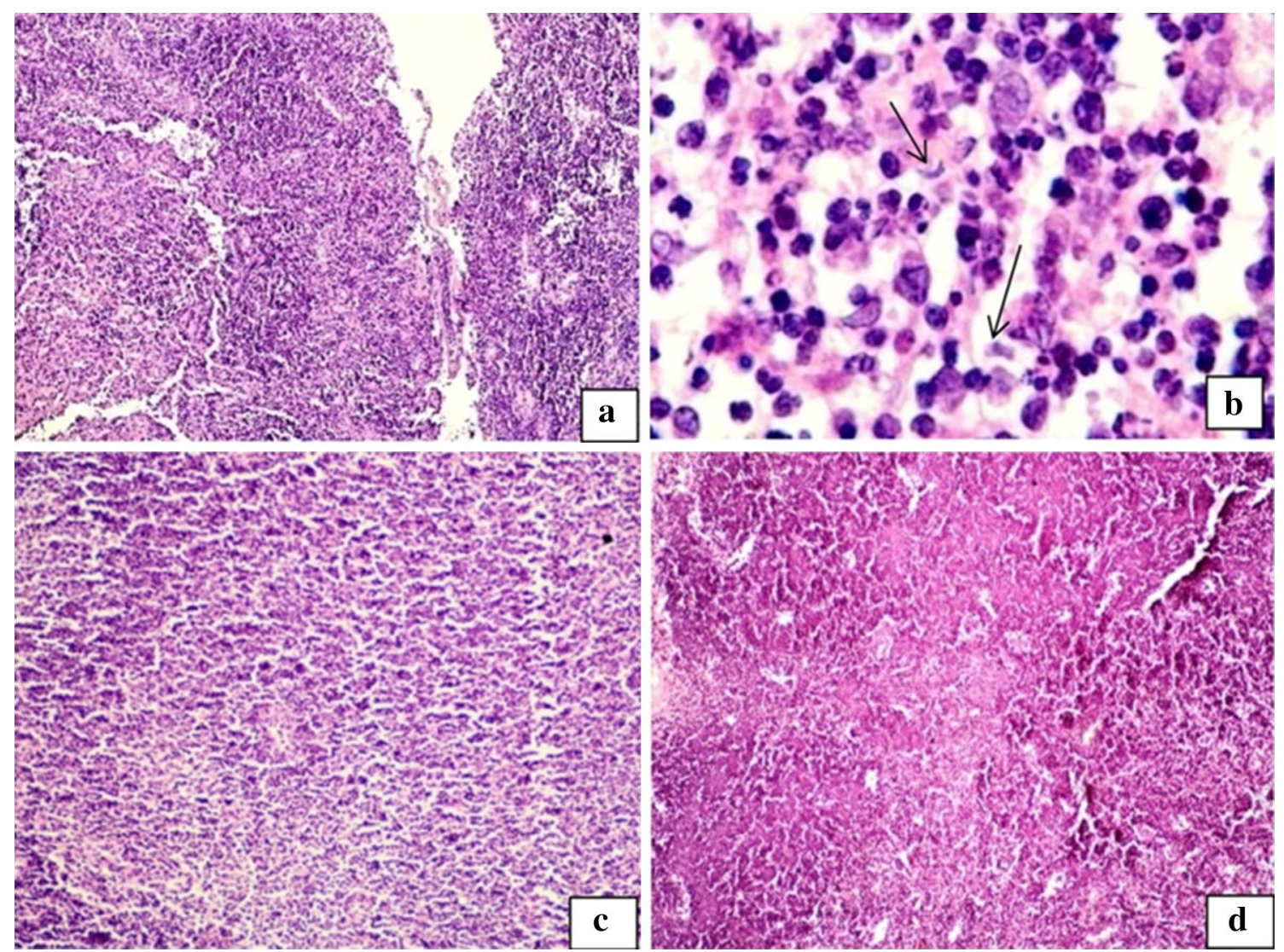

Fig. 6 MLNs sections in the studied subgroups. a MLN section of virulent RH-infected subgroup (Id) showing normal nodal architecture with lymphoid hyperplasia and prominent germinal center $(\mathrm{H} \& \mathrm{E}$ $\times 100$ ). b MLN section of virulent RH-infected subgroup (Id) showing small mature lymphocytes surrounding Toxoplasma tachyzoites $(\rightarrow$, $H \& E \times 1,000)$. $\mathbf{c}$ MLN section of CTLVECNS-vaccinated subgroup

character of chitosan may be attributed to the viscous nature of its solution creating an antigen depot. Over 20 years, chitosan was found to be a potent activator of macrophage and natural killer cells (Zaharoff et al. 2007).

In the present work, ionic gelation method using TPP as a crosslinking agent was used. The main advantage of this method is providing a mild condition without applying harmful organic solvents, heat or vigorous agitation that are damaging to sensitive proteins (Pan et al. 2002).

By SEM, enlargement of the nanospheres after vaccine encapsulation was evident and this is similar to the results of Cambridge et al. (2013) who reported a significant increase in the size of nanospheres after DNA encapsulation in a vaccine trial against Trachoma infection. TEM revealed spherical nanospheres, distinct and regular in shape but with a rough surface. This may be due to the presence of some antigenic material with a high molecular weight leading to their adsorption on the surface of the nanospheres during preparation (Gan and Wang 2007). challenged with virulent RH strain (IIa1) showing reactive changes in the form of follicle hyperplasia with germinal center formation $(\mathrm{H} \& \mathrm{E}$ $\times 100$ ). d MLN section of CTLV + FIA-vaccinated subgroup challenged with virulent RH strain (IIb1) showing lymphoid follicle hyperplasia with widening of the paracortical areas $(\mathrm{H} \& \mathrm{E} \times 100)$

These findings are in partial agreement with Cambridge et al. (2013) who revealed uniformity in particle size and a homogenous morphology of the formed nanoparticle.

The average hydrated particle size of vaccine encapsulated nanospheres was $221.5 \mathrm{~nm}$ with monomodal size distribution as revealed by Zetasizer instrument. These results were in partial agreement with Danesh-Bahreinni et al. (2011) using chitosan nanospheres as an adjuvant to Leishmania vaccine with a size range of $250-300 \mathrm{~nm}$.

XRD instrument was used to study the physical status (crystallinity) of both blank and vaccine-encapsulated nanospheres. Three prominent crystalline peaks were observed in both specimens indicating nanospheres stability. This may be attributed to the special characters of chitosan polymer, namely the presence of plenty of hydroxyl and amino groups forming strong intra/inter molecular hydrogen bonds (Saita et al. 2012).

When chitosan nanospheres were used as an alternative to FIA in combination with CTLV, the survival time, 


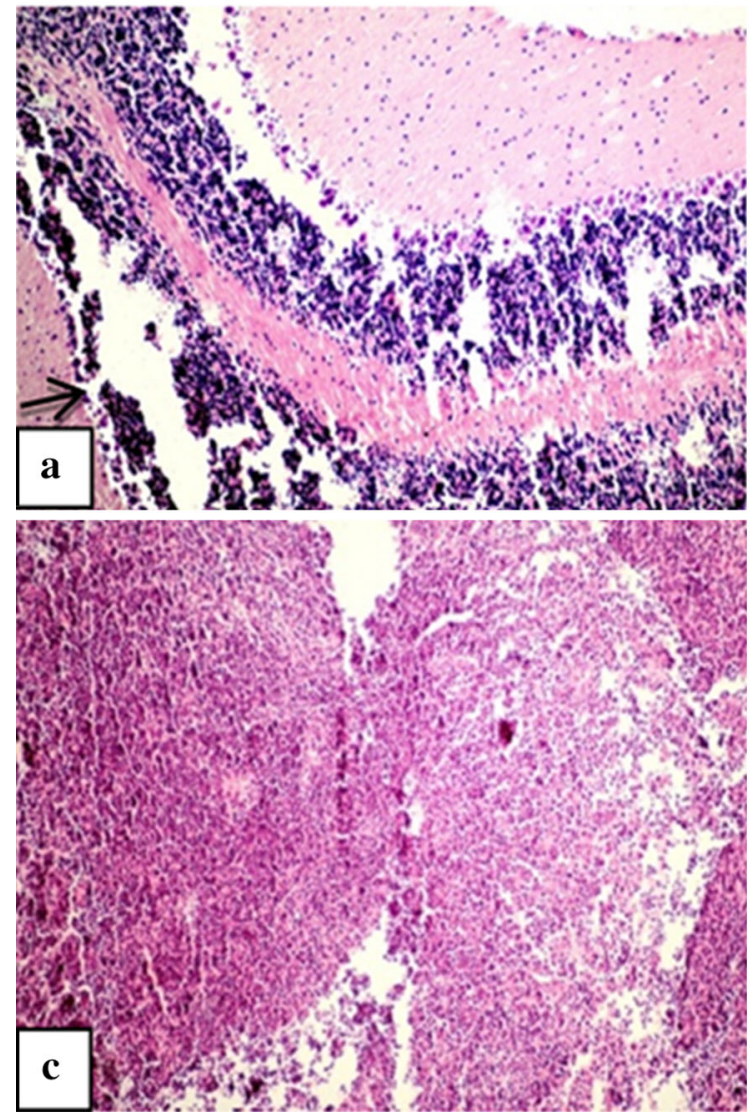

Fig. 7 Brain sections of the RH studied subgroups. a Brain section of virulent RH-infected subgroup (Id) showing heavy inflammatory infiltrates $(\rightarrow, H \& E \times 100)$. b Brain section of virulent $R H$-infected subgroup (Id) showing cortical tissue infiltered by a pseudocyst occupied by a single tachyzoite $(\rightarrow, H \& E \times 1,000)$. c Brain section of

humoral and cellular immune parameters increased, while the parasite count in different organs decreased. These changes were statistically significant when compared to the corresponding control subgroups.

In the acute infection model, a statistically significant increase in the survival time was noticed after being immunized with chitosan encapsulated nanospheres as compared to their corresponding control subgroup. Chitosan nanospheres were equally effective to FIA in increasing the survival time. These results are in agreement with other workers using temperature-sensitive mutant strain of T. gondii, ts-4 (Khan and Casciotti 1999), DNA vaccines (Yan et al. 2011), recombinant T. gondii nucleoside triphosphatehydrolase-II (Tan et al. 2011) recombinant surface tachyzoite antigen encapsulated in microparticles (Chuang et al. 2013), recombinant Toxoplasma gondii protein disulfide isomerase (rTgPDI) (Wang et al. 2013) and heat shock protein 70 gene (Kikumura et al. 2010). On the contrary, both Spencer et al. (2004) who used attenuated ts-4 strain with CPG as an adjuvant as well as Martin

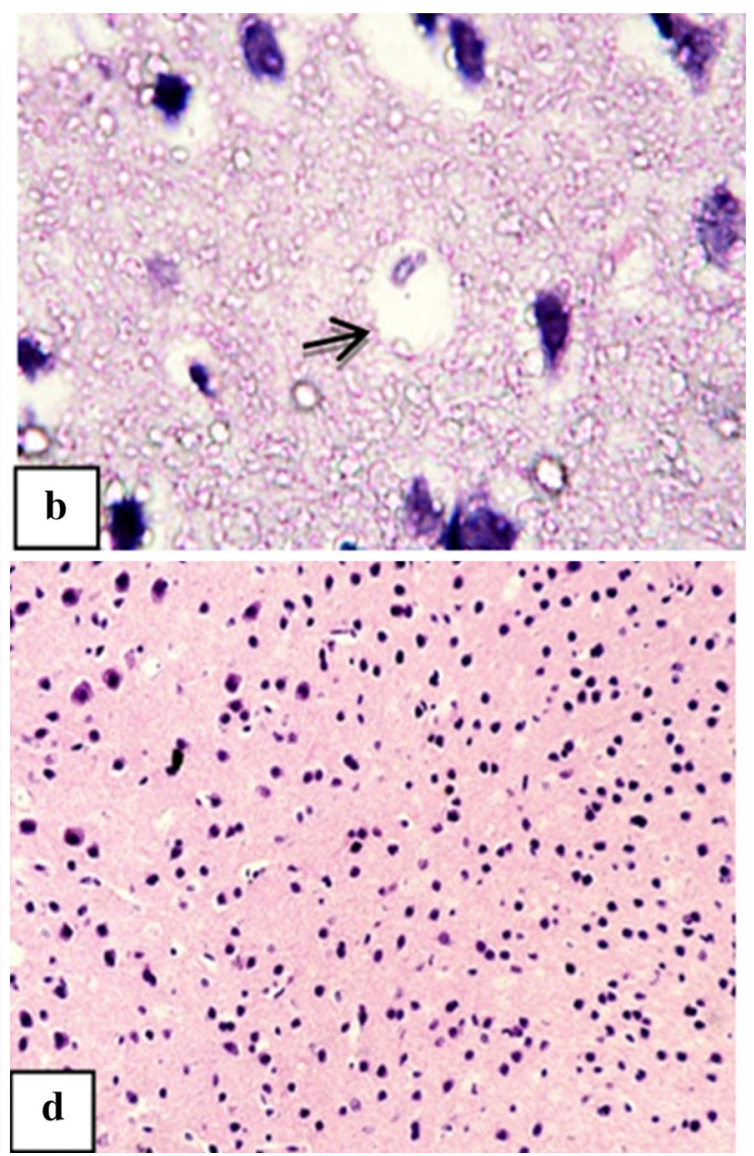

CTLVECNS vaccinated subgroup challenged with virulent RH strain (IIa1) showing normal brain tissue with no evident inflammatory infiltrates $(H \& E \times 100)$. d Brain section of CTLV + FIA vaccinated subgroup challenged with virulent RH strain (IIb1) showing increased cellularity with mild lymphocytic infiltrates $(H \& \mathrm{E} \times 200)$

et al. (2004) who used recombinant GRA4 or ROP2 vaccines with alum, reported no significant difference in survival time. In the chronic infection model, both types of vaccines succeeded to prolong the survival time. Chitosan nanospheres were less effective than FIA in increasing the survival time of the immunized mice. This is in agreement with other investigators using formalin fixed whole $T$. gondii tachyzoites (Krahenbuhl et al. 1972) or different antigens incorporated in liposomes (lipid based nanoparticles) (Elsaid et al. 1999).

Regarding the parasite count, a rapid dissemination of the parasite to the liver, spleen, MLNs and brain of the RH infected control subgroup was observed. This finding is in agreement with the studies done by El-Temsahy et al. (2002), Sibley et al. (2002) and Eissa et al. (2012). When chitosan or FIA were used as adjuvants to CTLV, a statistically significant reduction in the mean tachyzoite count in the different studied organs was observed with the highest reduction in the chitosan encapsulated vaccinated subgroups. This is in agreement with other workers who 

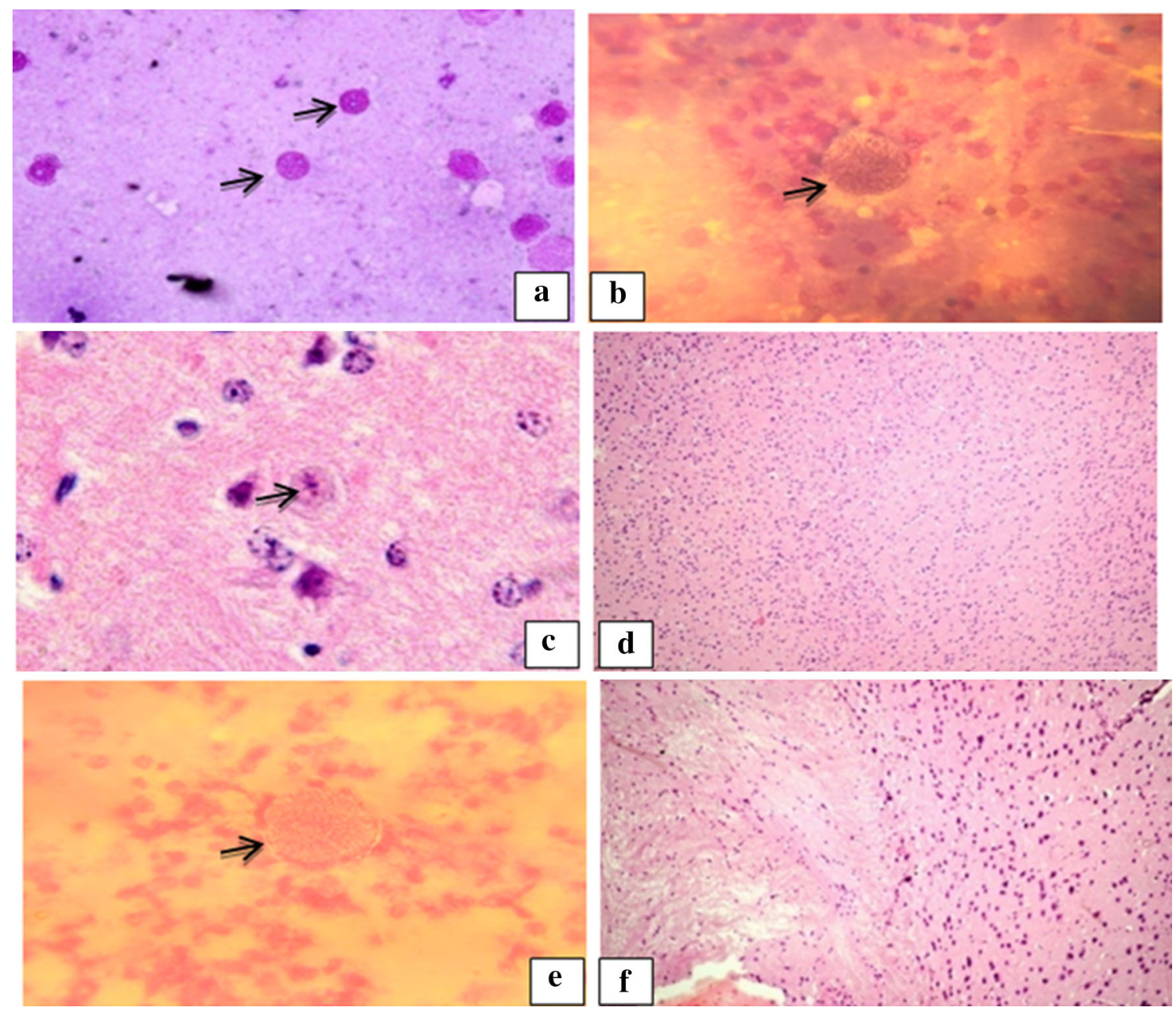

Fig. 8 Brain sections of the Me49 studied subgroups. a Brain section of avirulent Me49-infected subgroup (Ie) showing tissue cysts of Toxoplasma $(\rightarrow$, H\&E $\times 100)$. b Brain section of avirulent Me49infected subgroup (Ie) showing tissue cyst of Toxoplasma $(\rightarrow)$ surrounded by chronic inflammatory cells $(H \& E \times 400)$. c Brain section of CTLVECNS-vaccinated subgroup challenged with avirulent Me49 strain (IIa2) showing a Toxoplasma tissue cyst $(\rightarrow, \mathrm{H} \& \mathrm{E}$ $\times 200)$. d Brain section of CTLVECNS-vaccinated subgroup

reported reduction in the tachyzoites count after immunization of mice using CTLV + BCG (Eissa et al. 2012). In the chronic toxoplasmosis model, a statistically significant reduction in the tissue cyst count was found in both vaccinated subgroups. This is similar to the results of Zhang et al. (2013) using gene encoding calcium dependent protein kinase 3 in mice immunization, Makino et al. (2011) using Toxoplasma gondii heat shock protein 70 gene, Rabie et al. (2003) using heat shock protein 70 gene, heat shock protein 30 and SAG genes and Angus et al. (2000) using surface antigen incorporated within a plasmid vector in immunization of mice. On the other hand, a lower percentage of cyst reduction was reported by Martin et al. (2004) using granular and rhoptery proteins in combination with alum as a vaccine adjuvant. In the present work, the challenged with avirulent Me49 strain (IIa2) showing mild increase in the cellularity with no inflammatory cellular infiltrate $(\mathrm{H} \& \mathrm{E}$ $\times 100$ ). e Brain section of CTLV + FIA vaccinated subgroup challenged with avirulent Me49 strain (IIa2) showing a Toxoplasma tissue cyst $(\rightarrow, H \& E \times 200)$. f Brain section of CTLV + FIA vaccinated subgroup challenged with avirulent Me49 strain (IIa2) showing oedematous changes in the brain encroaching upon the parenchyma $(\mathrm{H} \& \mathrm{E} \times 100)$

highest brain cyst reduction was observed in the subgroup receiving the vaccine in combination with FIA and this may be related to the ability of the adjuvant to pass through the blood-brain barrier stimulating immune cells of the brain (Rabchevsky et al. 1999).

Histopathological changes in different organs of $\mathrm{RH}$ infected control subgroup showed inflammatory infiltrates, congestion, areas of lytic necrosis and tachyzoites within tissue sections. Similar changes were observed by Mady (2005). In animals injected with BCNS, the examined organs showed an enhanced immune response in the form of dilation of vascular spaces, hyperplasia of the lymphoid follicles and mild-moderate infiltration with inflammatory cells. While in the subgroup receiving FIA alone, severe degree of inflammation and necrosis were observed in 


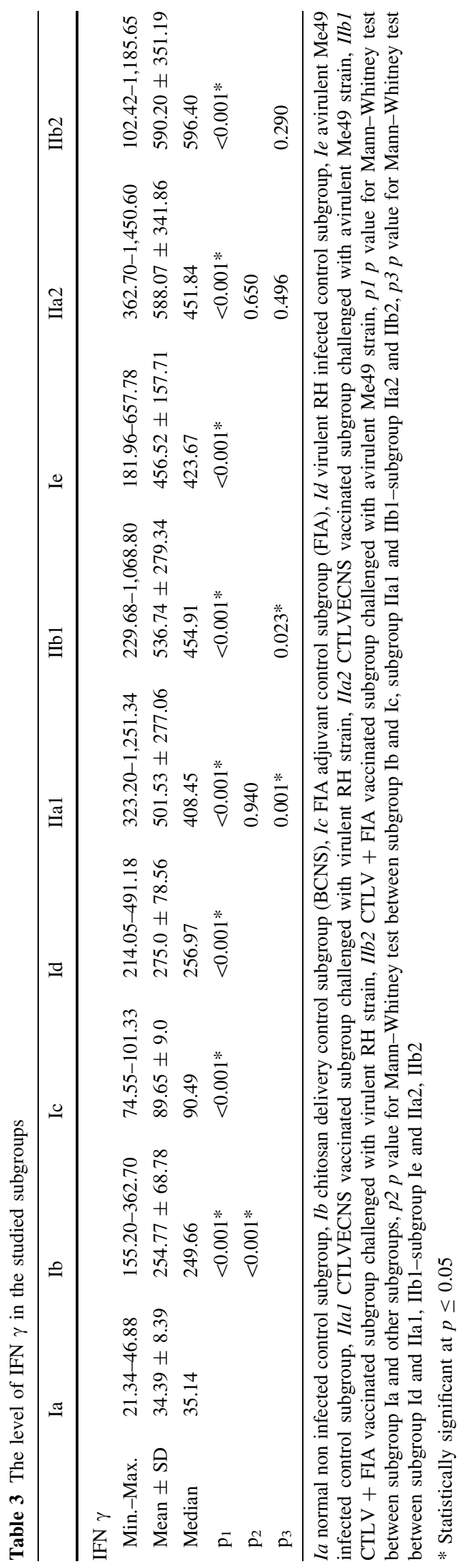

different organs especially the liver with ulceration at the injection site. The latter was also observed by Handman (2001) who reported severe pain in experimental mice with inflammation at the site of injection of FIA.

In vaccinated mice, all examined organs showed marked reduction in the severity and extent of inflammatory infiltrates, with very few foci of lytic necrosis within the parenchyma were observed with more reduction in the subgroup which received chitosan encapsulated vaccine. In liver tissue, changes observed were similar to those previously reported by Mady (2005) and Eissa et al. (2012). The findings of the splenic tissue examination were similar to those reported by Mady (2005) who used both autoclaved and lysate Toxoplasma vaccine and Shams El-Din (2013) who used lysate Toxoplasma and Influenza virus vaccine.

Histopathological examination of MLNs revealed lymphoid hyperplasia with a prominent germinal center in both infected and vaccinated subgroups. Tachyzoites were detected in the infected subgroup, however in the vaccinated infected subgroups lymphoid tissue was free from parasites. Similarly Mady (2005) and Eissa et al. (1990) noted the same histopathological changes with absence of parasites.

Brain tissue also showed heavy inflammatory infiltrates with pseudocysts in the cortical tissue of the brain of the RH infected control subgroup. These results are in agreement with other investigators who observed that non-suppurative encephalitis was the main picture of acute toxoplasmosis except for the presence of the parasite in the medulla (Escajadillo and Frenkel 1991; Mady 2005). As regards chronic Toxoplasma infection, brain cysts were detected, which is supported by Bakal and Veld (1979) and McLeod et al. (1988). Following vaccination with CTLV either within chitosan nanospheres or in combination with FIA, the severity and the extent of the inflammatory response were markedly reduced.

As protective immunity induced by $T$. gondii vaccine is complex and involves lots of elements, in this study both cellular and humoral immune responses were measured.

A statistically significant increase in the mean level of IFN $\gamma$ was reported in vaccinated challenged subgroups either in acute or chronic toxoplasmosis. Chitosan was equally effective to FIA in enhancing the vaccine immune response. This is in agreement with Zaharoff et al. (2007) who used $\beta$-galactosidase vaccine in either chitosan solution or FIA and reported no significant difference between them. A significant increase in IFN $\gamma$ level was also obtained by Meng et al. (2012), Chuang et al. (2013), Zhao et al. (2013a) and Cong et al. (2013).

In order to study the whole immunogenic picture of both vaccine and adjuvants, the humoral immune response represented by anti-Toxoplasma IgG and IgM were measured in serum samples of all studied subgroups. Although 
Table 4 The level of anti-Toxoplasma $\operatorname{IgG}$ in the studied subgroups

\begin{tabular}{|c|c|c|c|c|c|c|c|c|c|}
\hline & Ia & $\mathrm{Ib}$ & Ic & Id & IIa1 & IIb1 & $\mathrm{Ie}$ & $\mathrm{IIa} 2$ & IIb2 \\
\hline IgG level & 1 & 2 & 3 & 6 & 7 & 8 & 9 & 10 & 11 \\
\hline Min. & 0.16 & 0.16 & 0.14 & 0.15 & 0.46 & 0.54 & 0.42 & 0.61 & 0.58 \\
\hline Max. & 0.21 & 0.21 & 0.20 & 0.32 & 0.99 & 0.86 & 0.77 & 0.96 & 1.16 \\
\hline Mean & 0.18 & 0.19 & 0.19 & 0.23 & 0.65 & 0.67 & 0.57 & 0.72 & 0.74 \\
\hline SD & 0.02 & 0.02 & 0.02 & 0.04 & 0.16 & 0.11 & 0.10 & 0.12 & 0.16 \\
\hline Median & 0.18 & 0.18 & 0.19 & 0.23 & 0.65 & 0.65 & 0.58 & 0.65 & 0.71 \\
\hline $\mathrm{p}_{1}$ & & 0.495 & 0.069 & $0.004 *$ & $<0.001^{*}$ & $<0.001^{*}$ & $<0.001^{*}$ & $<0.001 *$ & $<0.001 *$ \\
\hline $\mathrm{p}_{2}$ & & 0.427 & & & 0.623 & & & 0.596 & \\
\hline $\mathrm{p}_{3}$ & & & & & $<0.001 *$ & $<0.001^{*}$ & & $0.010^{*}$ & $0.005^{*}$ \\
\hline
\end{tabular}

Ia normal non infected control subgroup, $I b$ chitosan delivery control subgroup (BCNS), Ic FIA adjuvant control subgroup (FIA), Id virulent RH infected control subgroup, Ie avirulent Me49 infected control subgroup, IIal CTLVECNS vaccinated subgroup challenged with virulent RH strain, IIa2 CTLVECNS vaccinated subgroup challenged with avirulent Me49 strain, IIb1 CTLV + FIA vaccinated subgroup challenged with virulent RH strain, IIb2 CTLV + FIA vaccinated subgroup challenged with avirulent Me49 strain, $p 1 p$ value for Mann-Whitney test between subgroup Ia and other subgroups, $p 2 p$ value for Mann-Whitney test between subgroup Ib and Ic, subgroup IIa1 and IIb1-subgroup IIa2 and IIb2, $p 3 p$ value for Mann-Whitney test between subgroup Id and IIa1, IIb1-subgroup Ie and IIa2, IIb2

* Statistically significant at $p \leq 0.05$

Table 5 The level of anti-Toxoplasma IgM in the studied subgroups

\begin{tabular}{|c|c|c|c|c|c|c|c|c|c|}
\hline & Ia & $\mathrm{Ib}$ & Ic & Id & IIa1 & IIb1 & $\mathrm{Ie}$ & IIa2 & IIb2 \\
\hline \multicolumn{10}{|l|}{$\operatorname{IgM}$} \\
\hline Min. & 0.18 & 0.17 & 0.19 & 0.16 & 0.30 & 0.33 & 0.18 & 0.32 & 0.37 \\
\hline Max. & 0.21 & 0.29 & 0.23 & 0.48 & 1.17 & 0.90 & 0.50 & 0.57 & 0.45 \\
\hline Mean & 0.19 & 0.21 & 0.21 & 0.32 & 0.44 & 0.44 & 0.38 & 0.41 & 0.40 \\
\hline SD & 0.01 & 0.04 & 0.02 & 0.12 & 0.26 & 0.17 & 0.10 & 0.09 & 0.03 \\
\hline Median & 0.19 & 0.20 & 0.21 & 0.33 & 0.36 & 0.40 & 0.37 & 0.37 & 0.39 \\
\hline $\mathrm{p}_{1}$ & & 0.544 & 0.111 & 0.082 & $<0.001^{*}$ & $<0.001^{*}$ & $0.002 *$ & $<0.001^{*}$ & $<0.001^{*}$ \\
\hline $\mathrm{p}_{2}$ & & 0.448 & & & 0.364 & & & 0.427 & \\
\hline $\mathrm{p}_{3}$ & & & & & 0.307 & 0.130 & & 0.880 & 0.289 \\
\hline
\end{tabular}

Ia normal non infected control subgroup, $I b$ chitosan delivery control subgroup (BCNS), $I c$ FIA adjuvant control subgroup (FIA), $I d$ virulent RH infected control subgroup, Ie avirulent Me49 infected control subgroup, IIal CTLVECNS vaccinated subgroup challenged with virulent RH strain, IIa2 CTLVECNS vaccinated subgroup challenged with avirulent Me49 strain, IIb1 CTLV + FIA vaccinated subgroup challenged with virulent RH strain, IIb2 CTLV + FIA vaccinated subgroup challenged with avirulent Me49 strain, $p 1 p$ value for Mann-Whitney test between subgroup Ia and other subgroups, $p 2 p$ value for Mann-Whitney test between subgroup Ib and Ic, subgroup IIa1 and IIb1-subgroup IIa2 and IIb2, $p 3 p$ value for Mann-Whitney test between subgroup Id and IIa1, IIb1-subgroup Ie and IIa2, IIb2

* Statistically significant at $p \leq 0.05$

the role of $\mathrm{IgG}$ antibodies in anti-Toxoplasma immunity is unclear, a general assumption has been proposed that serum IgG antibodies play a partial role in the prevention of secondary $T$. gondii infection (Kang et al. 2000; Johnson and Sayles 2002). A highly significant increase in the level of anti-Toxoplasma IgG was detected in vaccinated challenged subgroups whether in acute or chronic infection with no significant difference between chitosan and FIA. This result is supported by Tappeh et al. (2013) and Zhao et al. (2013a, b). Wang et al. (2013) and Yan et al. (2011) reported a marked increase in the mean level of IgG. The marked increase in the level of IgG in these studies may be attributed to the different types of antigens used for coating the ELISA microplate.
As regards, anti-Toxoplasma specific IgM antibodies, early appearance on the fifth day post infection was detected in RH infected control subgroup which is supported by Filisetti and Candolfi (2004), who reported its appearance at the end of the first week following infection. On the other hand, sacrificed mice infected with Me49 strain showed an increase in the mean level of anti-Toxoplasma IgM in comparison to the normal control and this is similar to results obtained by Gatkowska et al. (2008). As regards vaccinated challenged subgroups either in acute or chronic toxoplasmosis, no significant increase in the level of IgM in comparison to their corresponding control was detected. This is in agreement with Elsaid et al. (1999) using liposomes as adjuvant, who suggested that the 
vaccine used stimulated only cell-mediated immunity, but not humoral immunity.

In conclusion, this study showed that chitosan nanospheres were equally effective to FIA in enhancing the efficacy of Toxoplasma lysate vaccine. The effectiveness was demonstrated by the delayed death of vaccinated mice following challenge either with virulent $\mathrm{RH}$ or avirulent Me49 strains, the significant decrease in parasite density in different organs, the significant increase in the humoral and cellular immune response (IgG and IFN $\gamma$ ) with a marked reduction of pathological changes in the different organs. However chitosan nanospheres were superior to FIA due to their cost effective preparation and much less necrotic changes induced in the studied organs. In addition, the success of chitosan polymer as an alternative to commonly used adjuvants paves the way for the use of other newly developed polymers to be used in the field of vaccine development.

Acknowledgments We are grateful to Professor Ashraf Brakat, Department of Epidemiology and Zoonotic Diseases, National Research Center, Doki, Giza, Egypt for providing the avirulent Me49 Toxoplasma strain. Special thanks to Professor Rafaat Shaban, Epidemiology and Zoonotic Diseases, National Research Center, Doki and Dr. Mona Mohamed Tolba, Lecturer in Medical Research Institute for their assistance in ELISA technique. The technical support of Mrs. Neamat Ahmed Hassan is greatly appreciated.

Conflict of interests We declare that the authors have no conflict of interest.

\section{References}

Abu-Madi AM, Al-Molawi N, Behnke MJ (2008) Seroprevalence and epidemiological correlates of Toxoplasma gondii infections among patients referred for hospital-based serological testing in Doha, Qatar. Parasites Vectors 1:3-9

Angus CW, Klivington-Evans D, Dubey JP, Kovacs JA (2000) Immunization with a DNA plasmid encoding the SAG1 (P30) protein of Toxoplasma gondii is immunogenic and protective in rodents. J Infect Dis 181:317-324

Araujo FG, Phillippe P, Teri L, Remington SJ (1992) Activity of clarithromycin alone or in combination with other drugs for treatment of murine toxoplasmosis. Antimicrob Agent Chemother 36(11):2454-2457

Bakal PM, Veld N (1979) Response of white mice to inoculation of irradiated organisms of the Toxoplasma strain RH. Z Parasitenkd 59:211-217

Black MW, Boothroyd JC (2000) Lytic cycle of Toxoplasma gondii. Microbiol Mol Biol Rev 64:607-623

Cambridge CD, Singh SR, Waffo AB, Fairley SJ, Dennis VA (2013) Formulation, characterization, and expression of a recombinant MOMP Chlamydia trachomatis DNA vaccine encapsulated in chitosan nanoparticles. Int J Nanomed 8:1759-1771

Chan YH (2003) Biostatistics 102: quantitative data-parametric \& non-parametric tests. Singap Med J 44(8):391-396

Chuang SC, Ko JC, Chen CP, Du JT, Yang CD (2013) Induction of long lasting protective immunity against Toxoplasma gondii in
$\mathrm{BALB} / \mathrm{c}$ mice by recombinant surface antigen 1 protein encapsulated in poly(lactide-co-glycolide) microparticles. Parasites Vectors 6:34

Cong H, Zhang M, Xin Q, Wang Z, Li Y, Zhao Q et al (2013) DNA vaccine encoding SAG1/SAG3 with $A 2 / B$ subunit of cholera toxin as a genetic adjuvant protects $\mathrm{BALB} / \mathrm{c}$ mice against Toxoplasma gondii. Parasites Vectors 6:63

Danesh-Bahreinni MA, Shokri J, Samiel A, Kamali-Sarvestani E, Barzegar-Jalali M, Mohmmadi-Samani S (2011) Nanovaccine for leishmaniasis; preparation of chitosan nanoparticles containing Leishmania superoxide dismutase and evaluation of its immunogenicity in BALB/C mice. Int J Nanomed 6:835-842

Drury RAB, Wallington EA (1980) Carleton's Histological technique, 5th edn. Oxford University Press, Oxford

Eissa MH, Antonious SN, Salama MMI, Fikry AA, Morsy TA (1990) Histopathological studies of acute, chronic and congenital infections of toxoplasmosis in mice. J Egypt Soc Parasitol 20:805-816

Eissa MM, El-Azzouni MZ, Mady RF, Fathy FM, Baddour NM (2012) Initial characterization of an autoclaved Toxoplasma vaccine in mice. Exp Parasitol 131(3):310-316

Elsaid MMA, Vitor RWA, Frézard FJG, Martins MS (1999) Protection against Toxoplasmosis in mice immunized with different antigens of Toxoplasma gondii incorporated into liposomes. Mem Inst Oswaldo Cruz 94(4):485-490

El-Temsahy M, El-Kerdany ED, Abou-Shamaa AM (2002) Study of the role of antioxidants in experimental toxoplasmosis. J Med Res Inst 23:59-69

Escajadillo A, Frenkel J (1991) Experimental toxoplasmosis and vaccine tests in Aotus monkeys. Am J Trop Med Hyg 44(4):382-389

Filisetti D, Candolfi E (2004) Immune response to Toxoplasma gondii. Ann Ist Super Sanita 40(1):71-80

Gan Q, Wang T (2007) Chitosan nanoparticles as protein delivery carrier-systematic examination of fabrication conditions for efficient loading and release. Colloids Surf B 59:24-34

Garcia JL, Gennari SM, Navarro IT, Machado RZ, Sinhorini IL, Freire RL et al (2005) Partial protection against cysts formation in pigs vaccinated with crude rhoptery proteins of Toxoplasma gondii. Vet Parasitol 129:209-217

Gatkowska J, Gasior A, Kur J, Dlugonska H (2008) Determination of the value of Toxoplasma recombinant ROP2 and ROP4 antigen in experimental toxoplasmosis. Exp Parasitol 118:266-270

Handman E (2001) Leishmaniasis: current status of vaccine development. Clin Microbiol Rev 14:229-243

Hermes G, Ajika JW, Kelly KA, Mui E, Roberts F, Kasza K et al (2008) Neurological and behavioral abnormalities, ventricular dilatation, altered cellular functions, inflammation, and neural injury in brains of mice due to common, persistent, parasitic infection. J Neuroinflamm 5:48

Ismael AB, Dimier-Poisson I, Lebrun M, Dubremetz JF, Mevelec MN (2006) MIC1-3 knockout of Toxoplasma gondii is a successful vaccine against chronic and congenital toxoplasmosis in mice. J Infect Dis 194:1176-1183

Johnson LL, Sayles PC (2002) Deficient humoral responses underlie susceptibility to Toxoplasma gondii in CD4-deficient mice. Infect Immunol 70(1):185-191

Jongert E, Roberts CW, Gargano N, Förster-Waldi E, Petersen E (2009) Vaccines against Toxoplasma gondii: challenges and opportunities. Mem Inst Oswaldo Cruz Rio J 104(2):252-266

Kang H, Remington JS, Suzuki Y (2000) Decreased resistance of B cell-deficient mice to infection with Toxoplasma gondii despite unimpaired expression of IFN-gamma, TNF-alpha, and inducible nitric oxide synthase. J Immunol 164(5):2629-2634

Khan IA, Casciotti L (1999) IL-15 prolongs the duration of CD+8 T cell mediated immunity in mice infected with a vaccine strain of Toxoplasma gondii. J Immunol 16:34503-34509 
Kikumura A, Fang H, Mun HS, Uemura N, Makino M, Sayama Y et al (2010) Protective immunity against lethal anaphylactic reaction in Toxoplasma gondii-infected mice by DNA vaccination with $T$. gondii-derived heat shock protein 70 gene. Parasitol Int 59(2):105-111

Kirkpatrick LA, Feeney BC (2013) A simple guide to IBM SPSS statistics for version 20.0, student ed. Wadsworth, Cengage Learning, Belmont, California, p 115

Krahenbuhl JL, Ruskin J, Remington JS (1972) The use of killed vaccines in immunization against an intracellular parasite: Toxoplasma gondii. J Immunol 108:425-431

Leslie E, Geoffrey J, James M (1991) Statistical analysis. In: Interpretation and uses of medical statistics, 4th edn. Oxford Scientific Publications, Oxford, pp 411-416

Mady RFM (2005) Efficacy of killed vaccine against experimental toxoplasmosis. MS Thesis, Faculty of Medicine, Alexandria University, Alexandria

Makino M, Uemura N, Moroda M, Kikumura A, Piao LX, Mohamed RM et al (2011) Innate immunity in DNA vaccine with Toxoplasma gondii-heat shock protein 70 gene that induces DC activation and Th1 polarization. Vaccine 29(10): 1899-1905

Martin V, Supanitsky A, Echeverria PC, Litwin S, Tanos T, De Roodt AR et al (2004) Recombinant GRA4 or ROP2 protein combined with alum or the gra4 gene provides partial protection in chronic murine models of toxoplasmosis. Clin Diagn Lab Immunol 11:704-710

Martinez-Gomez F, Garcia-Gonzalez LF, Mondragon-Flores R, Bautista Garfias CR (2009) Protection against Toxoplasma gondii brain cyst formation in mice immunized with Toxoplasma gondii cytoskeleton proteins and Lactobacillus casei as adjuvant. Vet Parasitol 160:311-315

Mcleod R, Frenkel JK, Estes RG, Mack DG, Eisenhauer PB, Gibori G (1988) Subcutaneous and intestinal vaccination with tachyzoites of Toxoplasma gondii and acquisition of immunity to per oral and congenital Toxoplasma challenge. J Immunol 140(5): $1632-1637$

Meng M, He S, Zhao G, Bai Y, Zhou H, Cong H et al (2012) Evaluation of protective immune responses induced by DNA vaccines encoding Toxoplasma gondii surface antigen 1(SAG1) and 14-3-3 protein in BALA/C mice. Parasites Vectors 5:273

Palatnik-de-Sousa CB (2008) Vaccines for leishmaniasis in the fore coming 25 years. Vaccine 26:1709-1724

Pan Y, Li YJ, Zhao HY, Zheng JM, Xu H, Wei G et al (2002) Bioadhesive polysaccharide in protein delivery system: chitosan nanoparticles improve the intestinal absorption of insulin in vivo. Int J Pharmacol 249(1-2):139-147

Rabchevsky AG, Degos JD, Dreyfus PA (1999) Peripheral injections of Freund's adjuvant in mice provoke leakage of serum proteins through the blood-brain barrier without inducing reactive gliosis. Brain Res 832:84-89

Rabie MM, Fumie A, Mei C, Hye-Seong M, Kazumi N, Belal US et al (2003) Induction of protective immunity by DNA vaccination with Toxoplasma gondii HSP70, HSP30 and SAG1 genes. Vaccine 21:2852-2861

Ravindran R, Bhowmick S, Das A, Ali N (2010) Comparison of BCG, MPL and Cationic liposomes adjuvant system in Leishmanial antigen vaccine formulations against murine leishmaniasis. BMC Microbiol 10:181

Saita K, Nagaoka S, Shirosaki T, Horikawa M, Matsuda S, Ihara H (2012) Preparation and characterization of dispersible chitosan particles with borate crosslinking and their antimicrobial and antifungal activity. Carbohydr Res 349:52-58

Shams El-Din SA (2013) Evaluation of vaccination with Toxoplasma gondii trophozoite lysate and influenza virus vaccine against experimental murine toxoplasmosis. Acta Parasitol Glob 4(3): $110-120$

Sibley LD, Mordue DG, Su C, Robben DM, Howe DK (2002) Genetic approach to study virulence and pathogenesis in Toxoplasma gondii. Philos Trans R Soc Lond B 357:81-88

Spencer JA, Smith BF, Guarino AJ, Blaghurn BL, Baker HJ (2004) The use of CPG as an adjuvant to Toxoplasma gondii vaccination. Parasitol Res 92:313-316

Stoicov C, Whary M, Rogers AB, Lee FS, Klucevesk K, Li HC et al (2004) Coinfection modulates inflammatory responses and clinical outcome of Helicobacter felis and Toxoplasma gondii infections. J Immunol 173(5):3329-3336

Tafaghodi M, Tabassi AS, Amiri N (2008) PLGA Nanospheres loaded with autoclaved Leishmania major (ALM) and CPGODN: preparation and in vitro characterization. Iran J Basic Med Sci 11(2):112-119

Tan F, Hu X, Luo FJ, Pan CW, Chen XG (2011) Induction of protective Thlimmune responses in mice by vaccination with recombinant Toxoplasma gondii nucleoside triphosphate hydrolase-II. Vaccine 29:2742-2748

Tappeh KH, Khorshidvand Z, Shahabi S, Mohammadzadeh H (2013) A novel adjuvant, mixture of alum and naltrexone, elicits humoral immune responses for excreted/secreted antigens of Toxoplasma gondii tachyzoites vaccine in balb/c murine Model. Turk Parazitol Derg 37(2):92-96

Tenter AM, Heckeroth AR, Weiss LM (2000) Toxoplasma gondii: from animals to humans. Int J Parasitol 30:1217-1258

Thiptara A, Kongkaew W, Bilmad U, Bhumibhamon T, Anan S (2006) Toxoplasmosis in piglets. Ann NY Acad Sci 1081:336-338

Tiyaboonchai W (2003) Chitosan nanoparticles: a promising system for drug delivery. Naresuan Univ J 11(3):51-66

Turner HJ (1983) Detection of soluble antigens of Toxoplasma gondii four-layer modification of an enzyme immunoassay. J Clin Microbiol 17(5):768-773

Wang HL, Li YQ, Yin LT, Meng XL, Guo M, Zhang JH (2013) Toxoplasma gondii protein disulfide isomerase (TgPDI) is a novel vaccine candidate against toxoplasmosis. PLoS One 8(8):e70884

Yan HK, Yuan ZG, Petersen E, Zhang X, Zhou DH, Liu Q et al (2011) Toxoplasma gondii: protective immunity against experimental toxoplasmosis induced by a DNA vaccine encoding the perforin-like protein 1. Exp Parasitol 128:38-43

Yap SG, Kersten TS, Ferguson DJ, Howe D, Susuki Y, Sher A (1998) Partially protective vaccination permits the development of latency in a normally virulent strain of Toxoplasma gondii. Infect Immunol 66(9):4382-4388

Zaharoff DA, Rogers CJ, Hance KW, Schlom J, Grenier JW (2007) Chitosan solution enhances both humoral and cell mediated immune responses to subcutaneous vaccination. Vaccine 25(11):2085-2094

Zhang NZ, Huang SY, Zhou DH, Chen J, Xu Y, Tian WP et al (2013) Protective immunity against Toxoplasma gondii induced by DNA immunization with the gene encoding a novel vaccine candidate: calcium-dependent protein kinase 3. BMC Infect Dis 13(1):512

Zhao G, Zhou A, Lu G, Meng M, Sun M, Bai Y et al (2013a) Identification and characterization of $T$. gondii aspartic protease as a novel vaccine. Parasites Vectors 14(6):175

Zhao G, Zhou A, Lu G, Meng M, Min S, Bai Y (2013b) Toxoplasma gondii cathepsin proteases are undeveloped prominent vaccine antigens against toxoplasmosis. BMC Infect Dis 13:207 